\title{
Multi Perceptional Optimality Matrix Based Web Service Reliability Model
}

\author{
B. Muruganantham ${ }^{1 *}$, K.Vivekanandan ${ }^{2}$ \\ ${ }^{1}$ Department of Computer Science and Engineering, SRM University, India. \\ 2 Department of Computer Science and Engineering, Pondicherry Engineering College, India. \\ *Corresponding author. Email: bmnantha23373@gmail.com \\ Manuscript submitted April 4, 2015; accepted July 18, 2015. \\ doi: 10.17706/jsw.10.9.1045-1055
}

\begin{abstract}
To receive an appropriate response, the Multi Perceptional Optimality Web Service Recommendation Algorithm is proposed focus the research towards a multi attribute monitor that discovers on the profitable and preferable services by creating a recommendation matrix utilizing the top listing services ranked in a chronological order. Reliability is one of the most important Quality criteria of Service paradigms that can determine the popularity of web services for the completion of business process. Web services are more prone to failure than traditional software system due to their loosely coupled architecture, dynamic discovery and runtime binding. The technical flow incorporates the services that are registered to universal description, discovery and integration (UDDI) by service providers and the service consumers will look up for services from UDDI for functional relevance. To rank the web services various methodologies are incorporated and the utilized matrix method approach is the prominent amongst the other techniques. But, it fails to define certain QoS parameters precisely and thus it cannot support complex QoS constraints in terms of composition. The postulates include manipulating the differences between Numbers of requests Vs Number of successful requests processed by the web service provider by utilizing the Multi Perceptional Optimality Web Service Recommendation Algorithm. The Existing methodologies emphasize on the process continues through coordination and combination of different services based on the ranking factors such as response time and cost to access the service. The Proposed methodologies incorporate Service Alive Percentile and attain more accuracy on the Web Service with proper reliability.
\end{abstract}

Key words: Web service composition, preferable services, profitable services, web service decision zone, WS invocation zone, WS recommendation zone.

\section{Introduction}

Web service architecture is an emerging technology that consumes multiple services with the same functionality. It builds up the distribution and independent service compositions based on reliable Quality of Service. Service interface, service discovery and service invocation are carried out using the on syntactic XML based standards such as WSDL, UDDI and SOAP. Standardized protocols receive service requests from the clients. Appropriate services are selected by identifying and coordinating the individual service components that meet the functional needs of the end user. Several service components consist of three entities the Service Provider, the Service Registry and the Service Consumer. With an escalating delivery of many service providers, the Web-service related techniques have become popular to improve the system 
integration and interaction. This is achieved to delinquently encompass to offer different ranking levels of the services in order to congregate the demand of dissimilar consumer groups. The measurement $n$ the server side is not sufficient for the service users, since the service availability metrics are supposed to be expected based on the success rate of service invocations (according to the "distinct position of instant" metric). But, the "distinct position of instant" metric have its possession of limitation.

The service does not have to provide a special operation to test the reachability of a host on an Internet Protocol using the ping command. In order to categorize and accommodate the exceptionality of failures and successes of service invocation, a novel availability metric has been emphasized by associating the QoS issues of composite services with the exceptionally probabilistic service response outcome and this method acts as an estimated approach. The fundamental suggestion of metric paradigm is that, the reliable service is consecutively running in the diverse End-to-End constraint and each category of individual web services [1], [2] has its own features of encountering failures. To overcome the conflicts of the stated scenario, a Recommendation Matrix model is introduced that proposes a novel estimation approach for reliable service that acquires the corresponding features of each composite and distributed web service.

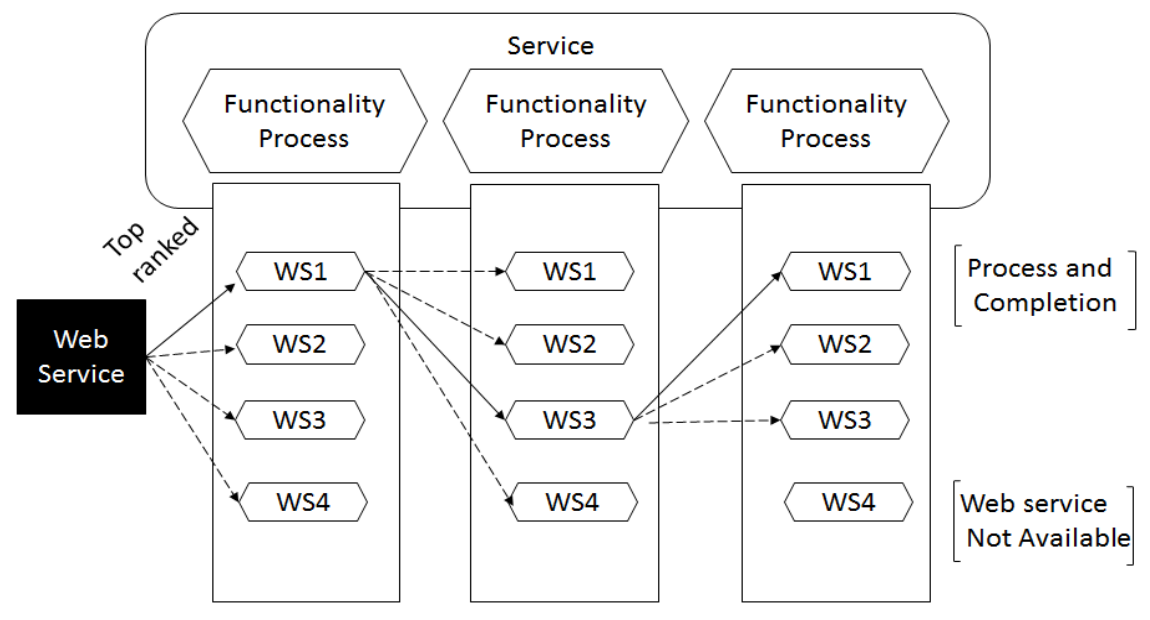

Fig. 1. Functionality of web service reliability.

In Fig. 1 based on a widespread set of web service discovery mechanisms in the monitored services, the encountered continuous successes are considered to be the most stringent service. At first, the web service client can encounter transient invocation failures, but still it can be successfully accessed from the next level of several invocations. On the other hand, the transient invocation successes and failures tend to occur at various time slots based on the requests made by the users evolved in each architectural type of reliable services. The web service selection is performed individually for each task and a service with the best score is chosen over the depiction of continuous failures in the services. Based on heuristic approach, the invocation records are composed of the web service client side network where the user investigates the apparent failures of the service. There are several methods in the service composition that ought to be generalized purely based on the user-side data and must be regarded as reliable web service factors.

\section{Real Time Scenario and Reviews}

\subsection{Real Time Scenario}

A discussion about a motivating example of the car manufacturing application is presented below to support with the proposed approach. This application is carried out by a composite service as illustrated in Fig. 2. The services involved in this car manufacturing application are:

- The Car Requirements Specification (CRS) service (Service Invocator 1) 
Used to receive the car manufacturing, customer order and to review the customer requirements,

- $\quad$ The Catalog of Ordered Items (COOI) service (Service Invocator 21)

Used to order the car components if the online store does not have all of it,

- $\quad$ The Credit Card Payment Processing (CCPP) service

Used to guarantee the payment by credit card, (Service Invocator 23)

- $\quad$ The Assembling Car Parts (AC) service (Service Invocator 3)

Used to ensure the car assembly once after the payment is done and the required components are available, and

- $\quad$ The Car Delivery (CD) service (Service Invocator 22)

Used to deliver the cars to the customer (provided either by National Packers \& Movers (CD-NPM) or Indian Car Transport (CD-ICT)). (Service Invocator 4)

When a user selects a composite service, the expectation of the reliability tends to be far above the good. Negative scenarios predominantly paid more attentions from the user's perspective. In the above stated example, the car manufacturing designer requires to know the likelihood of one of the two delivery services will succeed. Considering the service CD (NPM) (National Packers \& Movers) is not completed, then it must be possible for the service COOI (Catalog of ordered items) to undo its effects (for instance when the payment fails). These properties define what we call the transactional behavior of the service. This behavior is specified using a set of reliable services. In most cases the requirement varies according to the environment, and hence the transactional behavior will vary too. For instance, a designer may accept the failure of the CD (NPM) service in the initial context.

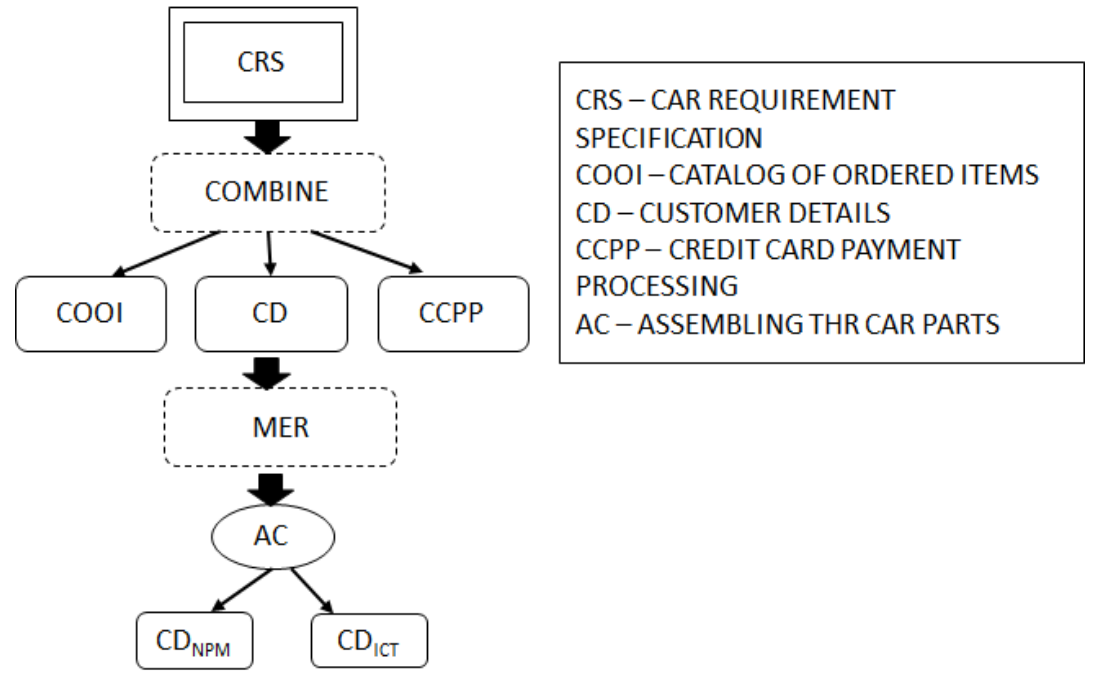

Fig. 2. A composite service for car manufacturing.

while he may not tolerate such a failure at an advanced stage. So the means of a reliable execution are tightly related to transactions and manufacturing designers' requirements. But, in order to confirm a reliable execution, it is necessary to ensure that a specified car service behavior is consistent with the set of selected services and the reliable transaction requirements. Coming back to the manufacturing example, it is easily noticed that since the categorization of ordered item service is not sure to complete, the payment service CCPP have to be compensatable (and it must be compensated when the COOI service fails). By analyzing this real time implementation, the reliable services are identified and provided to the customers at the preferable time based on the profitability of web service.

\subsection{Literature Review}

During the last few years, the issue of reliability based web service composition and selection has gained 
a lot of consideration by many researchers. To fulfill user's requirement and to achieve the reliable business process, composition of services can be used and it's attainable using an incremental optimal Suggestion technique [3] by expanding the search space for top-k possible composite services. A new hybrid bounded set technique [4], [5] which involves a hybrid model combining both paths based and state (Idle and Active) based approach is introduced in the service reliability area. In this technique, a centralized broker works as a coordinator invoking appropriate web services and the user's correctness of service execution can be analyzed through the integration of process interface constraint [6]. Based on their state and in case of nonavailability, a rollback mechanism to prefer an alternative path of web service methods invocation is integrated. Identifying and grouping the similar functionalities embossed in the composite services is much more interesting fact and this can be achieved by analyzing multiple factors such as response time, cost of service invocation, service availability and much more. Composite service skyline [7] technique provides an optimized solution on the dominance relationship among the available service providers vs. the best services offered. The proposed solution provides a filter mechanism in getting the best sky lined services instead of scanning through all the possible service compositions. This will provide a time effective solution for the web service composition arena. The preferable services can be identified using a generic fuzzy based query evaluation paradigm [8] which enables a services search and holds the tendency of combining with the existing services in fulfilling the business request. This can be achieved through parallel and serial configuration with a centralized backbone co-ordination. In terms of coordination, Byzantine fault tolerance provides an optimized solution by integrating with Kandula framework [9], [10] and it emphasize source ordering providence of different web service's incoming request. During the failure of web service discovery and invocation, Semantic-based service categorization [11] provides an optimal impact in case of process exit. This will provide an improved level of services and can be achieved through an ontological framework. In turn, a functional level of service categorization embedded with latent semantic indexing of services.

\section{Materials and Methods Combinatorial Availability Cum Reliable Rank Matrix Monitor}

\subsection{WS Recommendation Model}

Web services are more used as the primary chunk for creating inters and intra-enterprise business progressions. Such business progressions are created through the adaptation of several obtainable Web services in a rational order that satisfies certain necessities. Web services are intrinsically dynamic by themselves, and hence cannot be stable ever. It is in their very temperament to reveal the most recent edition of an upheaval or the job. There is no guarantee that the usual development of a service will not change its boundary, and therefore disconcert a previously working adaptation. Hence the question of discussion is their availability at the required time. The need for the web service recommendation model is to select the appropriate web service from more a number of available web services that possess similar functionality with invariant service provisions. It necessitates the requestor with the desired web service discovery as they may not be conscious of the relevant and favorable services available.

In the proposed scenario of the web service model, a recommendation model is supposed to service the user based on their requirements. The generic model of the web service recommendation is a layered approach of process that initially utilizes the web service requests from the user through the access point. An encountered incoming web service request is traversed to the process zone through the recommendation zone wherein the income request gets segregated into one of the two Selection criteria such as Preferable and Profitable Services. A Preferable Selection criterion service is selected to serve the user only by checking the availability, condition of the web service with respect to Inconsistencies, 
Parameter incompatibility, Time-Out, Incorrect services, SLA (Service Level Agreement) and irrespective of Cost wise/Distance wise/Delivery wise Manipulation. A Profitable Selection criterion service is selected to serve the user only by checking the availability, condition of the web service with respect to Cost wise/Distance wise/Delivery wise Manipulation irrespective of Inconsistencies, Parameter incompatibility, Time-Out, Incorrect services, SLA (Service Level Agreement). The Successive step towards the service selection is to process the request from the decision point to choose the Service provider that exactly satisfies the incoming request with the selection criterion. The Decision point layer decides upon selecting the backup service during the occurrence of web service failure. A combinatorial availability cum reliable rank matrix monitoring is proposed as the recommendation matrix designed by considering the user Request, Web Method compositions, Quality Of service component, Selection of services for providing an efficient response from the service provider. During the failure of web service, a handover to backup service is done by identifying the similar service provided by the web methods suitably matched in the combinatorial matrix or by issuing the same available service provider of the incoming request based on the similarity of the web method. A Log Based Maintenance is done with the Archiving concept with retention Period to analysis the previous request/response state for identifying the effectiveness of the service provider in the matrix monitor.

\subsection{Problem}

In the Traditional approach, the Web service recommendation system [12] concentrates on the same dictum of satisfying user's requests with the best available service. The review reports of the web service recommendation system articulates that the selection of the many number of available web service either by the rating based on user reputation metrics or by the Trust based metadata ontology information. The above stated traditional scenario of rating or ontological based service provision can only apply only if the signature of the user's previous requests is known to the recommendation engine to serve them the best with the rating analysis [1], [13], [14]. There could be no possible solution in the traditional approaches for initial stage of requests from the access points with many numbers of service providers with similar functionality. The main discomfort is that a rating based recommendation model cannot be more effective to the user with different preference and taste. The system provides the response only by considering the availability of the service provider that cannot serve the user's request effectively. The untrustworthiness of any of the component Web Services could lead to the failure or QoS deprivation of the acquired service, even if another component service happens to be reliable. Concentrating on the reliability and the availability of Web services is a demanding task, particularly identifying and managing errors and issues. Presenting a reliable Web Service is a judicious problem.

\subsection{Combinatorial Availability Cum Reliable Rank Matrix and Multi Perceptional Optimality Web Service Recommendation Algorithm}

The above matrix provides the percentile options for a particular service in the specific period. The matrix considers to have $\mathrm{x}$ and $\mathrm{y}$ coordinates as the Date and Service. The availability of the Service is measured with in terms of time with respect to the coordinates $\mathrm{x}$ and $\mathrm{y}$ taken as Date and Service. The resultant is the cumulative Percentile availability of the Service. Mathematically, it is stated as the Summation of the available time based Service with applicable Dates.

$$
{ }_{n} C_{p}=\sum T_{s}^{i=1}
$$

where $C_{p}$ is the cumulative Percentile, 
$T$ is the Time Component,

$i$ is the Date Component,

$S$ is the Service Component,

$n$ is the limited up to 100 transaction.

The second step forms the recommendation matrix. The matrix is arrived upon Multi Perceptional Optimality Web Service Recommendation Algorithm with weightage and Utilization Factors (UF) of the Web Service. Based on UF a service percentile is derived as per the below formula:

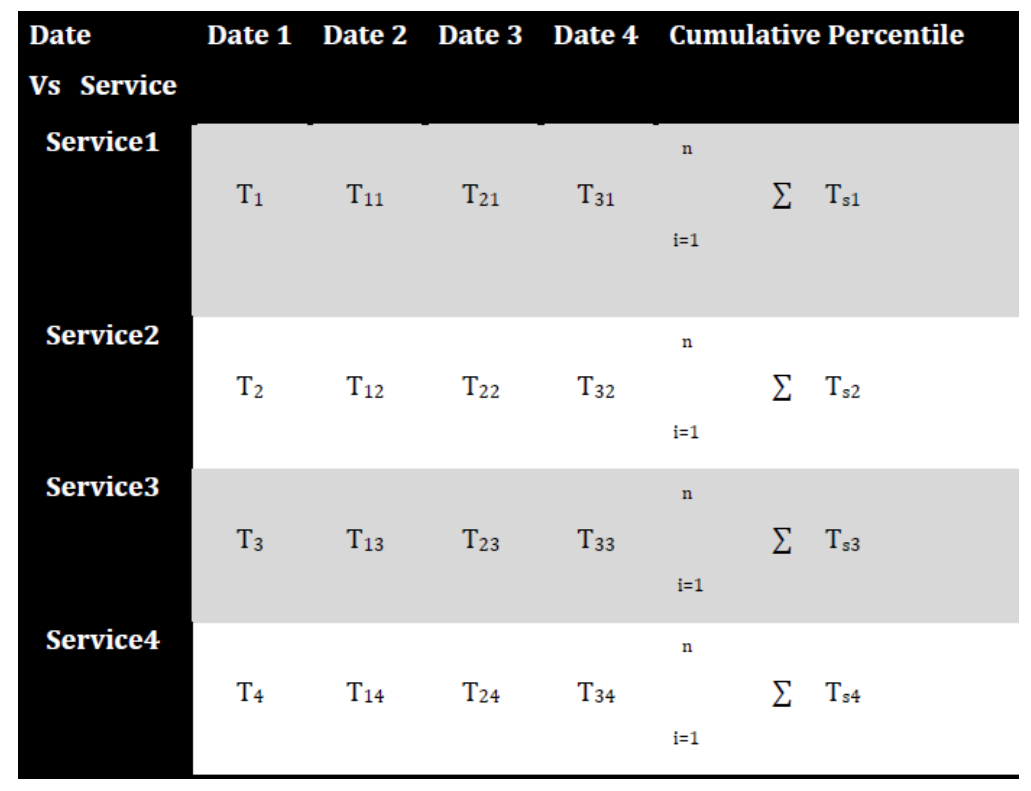

Fig. 3. Cumulative reliable rank matrix.

$$
U_{f}=\left(\frac{W_{r s} \times R P_{n}}{R S_{t}}\right) \times P_{a}+\left(\frac{W_{c} \times R P_{n}}{R S_{t}}\right)
$$

where $U_{f}$ is the utilization factor,

$W_{r s}$ is the weight of the response time

$W_{c}$ is the weight of the cost

$R P_{n}$ is the number of request processed

$R S_{t}$ is the total number of response sent

$P_{a}$ is the availability percentile

$U_{f}$ is a utilization factor based on behavioral factor of the request evaluates the description about the item requested by the user from the access point, availability of the service provider, Cost of the service, response time of the service. This factor is utilized as the key factor in service handover. $W_{r s}$ is the weight of the response time; this is factor gives the exact waiting time of the user after raising the request. $W_{c}$ is the weight of the cost with respect to time, the preferability and profitability is determined with this factor, $R P_{n}$ is the number of request processed and $R S_{t}$ is the total number of request sent are the status based factors of the web service. $P_{a}$ is the availability percentile, Percentile component is the combined measure 
of Availability of a service with respect to the Date of its availability, Time of its availability in the available Date and the type of the Service available in the particular Date and Time.

A $\log$ based Maintenance for and request limited to 100 transactions is made to achieve in the repository for analyzing the effectiveness of the Service Provider. A retention period $(x)$ is considered to be twice the retention period per log Maintenance.

\subsection{Pseudo Code}

Step 1: Service Consumer raises the Service Request from the access point

Step 2: Service Selection process for the Invoked Service Request

Step 3: Matrix Monitorer acquires the Utility Web Method allocation of the Service Provider

Step 4: Identification of Percentile Availability $P_{a}$ Of the service Provider.

Step 5: Identification of the Weight of Response Time $W_{r s}$ of Previous Responses given by the Service Provide.

Step 6: Identification of the Weight of Cost $W_{c}$ with respect to Time.

Step 7: Identification of the Number of Request Processed $R P_{n}$ of Previous Requests given by the Service Provide.

Step 8: Identification of the total number of response sent $R S_{t}$ of Previous Requests given by the Service Provider.

Step 9: Calculating the Product of Weight Of Response Time $W_{r s}$ of Previous Responses and Number of Request Processed $R P_{n}$ of Previous Requests given by the Service Provide per Total Number Of Response sent $\mathbf{R S}_{\mathbf{t}}$ of Previous Requests.

Step 10: Multiplying the Resultant of Step 9 with the Percentile Availability $P_{a}$ of the service Provider.

Step 11: Calculating the Product of Weight Of Cost $W_{c}$ with respect to Time and Number of Request Processed $R P_{n}$ of Previous Requests given by the Service Provide per Total Number Of Response sent $R S_{t}$ of Previous Requests.

Step 12: Perform Summation of Step 10 and Step 11

Step 13: Store the Resultant as utilization factor $U_{f}$.

\subsection{Solution}

To solve the issue of the problem stated above and to design a reliable web service with perfect recommendation zone that overcomes the issues of immaturity standard service, performance consideration in terms of delay in response time, Service trust, Failure handover, Issuing appropriate service, a matrix based recommendation zone is designed. The matrix named Combinatorial Availability cum Reliable Rank Matrix is proposed as the designed model of the recommendation zone of the web service. It is the matrix that has been designed with the utilization factor, Availability with respect to time, date and the service that acquires it as a combinational matrix. Each Utilization Factor defines the available service providers' ability with the response time, cost it requires to complete the request, to resolve an incoming user's request in the form of web methods. The designed combinatorial matrix is used by the utilization factor to serve the user's request from the access point.

In the WS Recommendation model layered approach, the request of the user's from the access point is acquired. The selection process is carried out to identify the type of the requirement of the service to be profitable or preferable. Based on the Selection criterion, the Utilization factor is allocated to the incoming request in the recommendation zone. The WS recommendation zone comprises of many numbers of service provider with similar functionality and invariant service. The Number of available providers needed is decided to serve the income request of the user in the decision zone. According to the required count of the service provider to process the request, the other measures such as availability, Time, Date with selection 
criterion is taken as the Percentile component to the monitor matrix. The input is translated in the form of web methods to serve the request with the best suitable service provider applicable in the matrix monitor. The response is then provided to the user from the service provider. Providers as per the process completion with perfect finish over handover strategies.

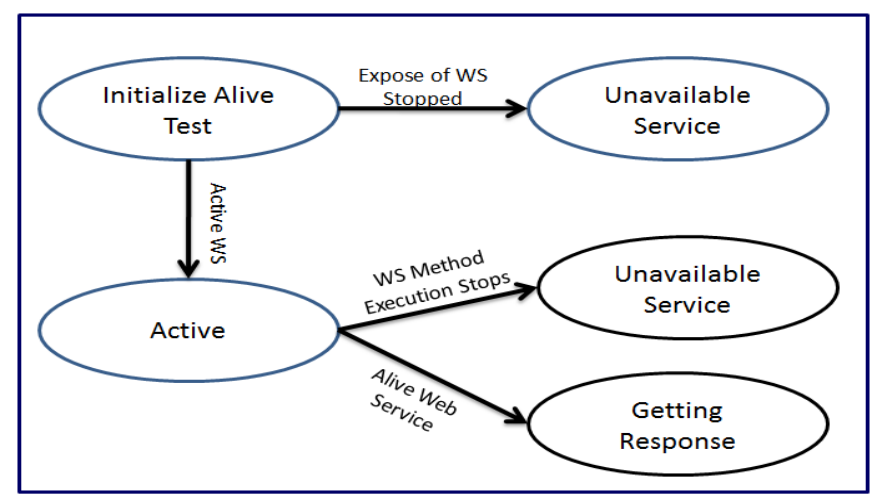

Fig. 4. Web service states.

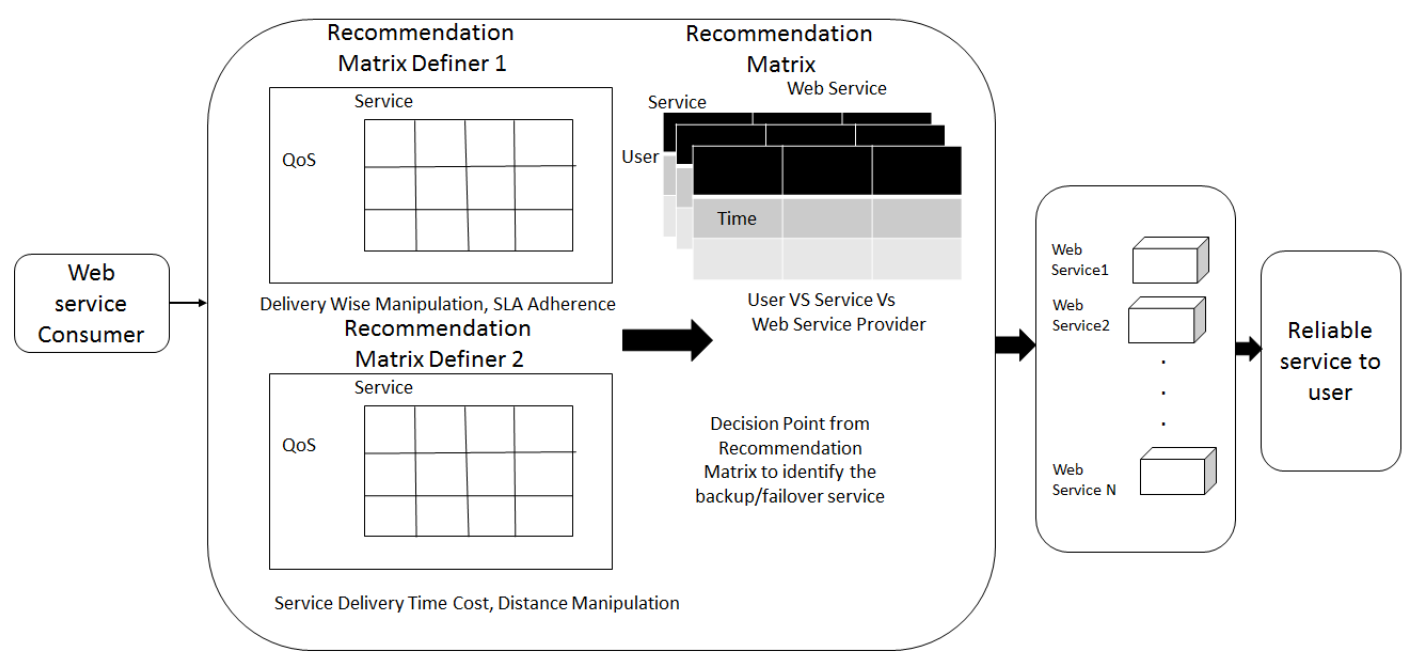

Fig. 5. Service availability percentile with respect to time.

The Web service states are considered by the matrix monitorer in a life cycle event as follows in the Fig. 4 . The web service has various evaluations as indicated. Each and every web service, that satisfies the user request, has an initial state and is exposed to service users. According to the first step of Combinatorial Availability cum Reliable Rank Matrix Monitorer, the user selects a set of web services depending on its active state and also along with the decision made from a set of reliable services with the utilization factors by the decision zone. Monitored in the Fig. 5 stated above Creates a replica of performance under failure of multi-tier Web Services as it is an essential and the major step to build a reliable Web Service. With a mechanism of failure recovery, other alternate services must be in place to avoid chaos. So a decision point is reached based on availability to decide and select from the list of matching services. The matching web service is identified by using the translated web methods of the user request framed before applying the request to the utilization factors previously in the request invocation. The equivalent available service or the similar alternate service provider is chosen by the web method equivalence from the monitor matrix as it already has the set applicable, available and the number of service provider to serve the income request with the calculated utilization factor of the user's request. 
The effectiveness of the hand over service by the service provider is calculated for further new utilization factor calculation being done for new requests. This overcomes the mechanism utilized in the traditional model such as fault tolerance calculated to recover the failed web service to serve again as it increases the waiting response time of the user from the access point.

\section{Results and Discussion}

\subsection{Performance Evaluation}

Table. 1. Web Services and Ports 494

\begin{tabular}{|l|l|}
\hline WebService Name & Port Number \\
\hline \multirow{4}{*}{ AdditionalSearchWebService } & 1421 \\
\cline { 2 - 2 } & \\
\hline \multirow{2}{*}{ RecommendationService } & 11362 \\
\cline { 2 - 2 } & 11494 \\
\hline \multirow{3}{*}{ SearchWebService } & 1408 \\
\hline & 11777 \\
\cline { 2 - 2 } Login & 1515 \\
\cline { 2 - 2 } & \\
\hline
\end{tabular}

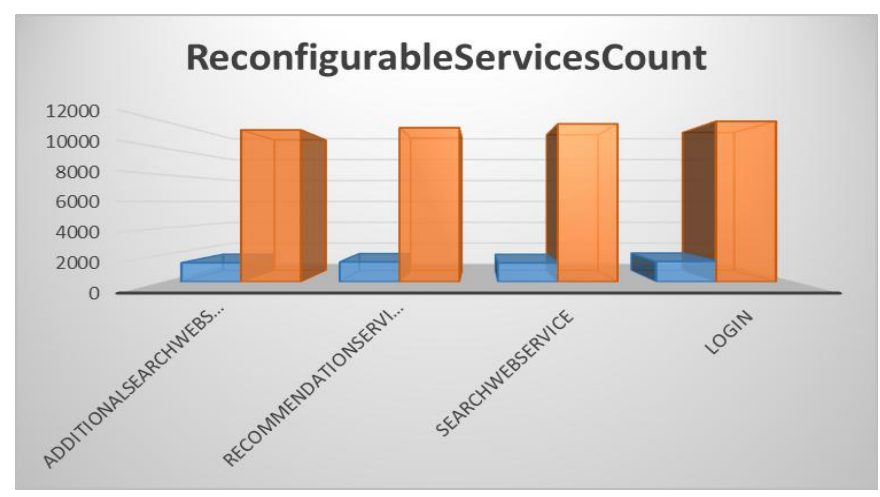

Fig. 5. a). reconfigurable web service.

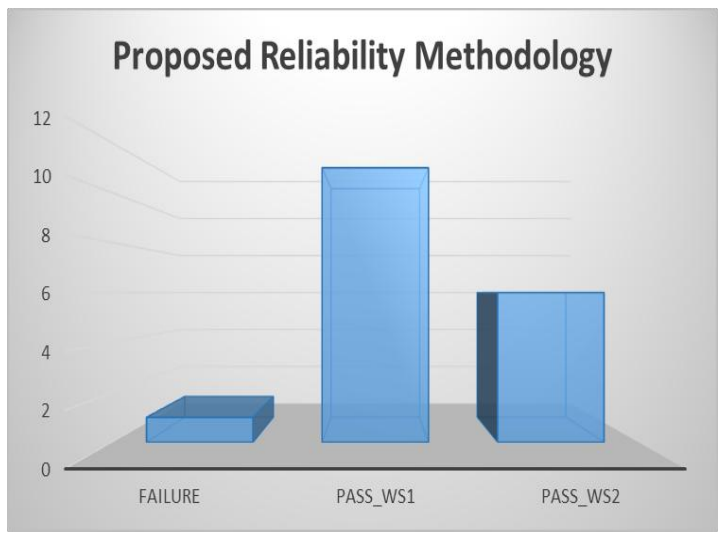

Fig. 5. b). Proposed system reliability methodology.

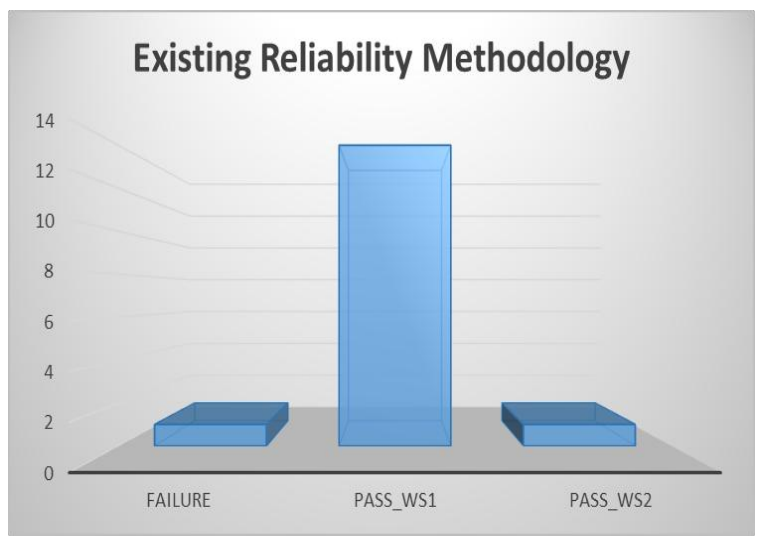

Fig. 5. c). Existing system reliability methodology. 
The above tabulation represents the Designed Web service name and the port number in the experimental implementation. Each Web service encompasses two ports to make the reconfigurable service efficient to satisfy multiple user requests absolutely.

Fig. 5. a). Performance Evaluation of Web service with respect to reconfigurable web service. 5.2 Comparison of Proposed system with respect to reliability Methodology.5.3 Comparison of Existing system with respect to reliability Methodology

The Fig. 5. a). evaluates the performance of web service designed as represented in the tabulation above. This showcases the reconfigurable web service response to the request count that acquired in the graphical representation. The Fig. 5. b). evaluates the performance in terms of reliability measures of the proposed methodology for the successive and failure result. The Fig. 5. c). evaluates the performance in terms of reliability measures of the Existing methodology for the successive and failure result. Both Fig. 5.2 and Fig. 5.3 depicts that the proposed methodology handles more requests and provide a reliable service provision than the Existing methodology.

\section{Conclusion}

As a perceptive comprehensive algorithm Multi Perceptional Optimality Web Service Recommendation Algorithm for Combinatorial Availability cum Reliable Rank Matrix Monitorer can obtain a high-quality resultant in the area of web service reliability. The problem with the existing system of providing the service to the user request with the web service recommendation model is avoided. The generated matrices in the postulates have collective input source from the user request with the utility factors and generates the suitable number of service providers with their availabilities along with specific selections. Our Study and postulates with improved set of formulae and Matrixes found to be optimal option than the existing scenarios. This is experimentally proved for the effectiveness in the reliability of the Web service selection process.

\section{References}

[1] Raja, B. K., \& Kaliyamurthie, K. P. (2014), Trustworthy management for safety banking using light weight BFT method. International Journal of Inventions in Computer Science and Engineering, 1(2).

[2] Khushboo, R. S., \& Saravanan, T. (2014). Radio frequency technology for intelligent transportation system. International Journal of Inventions In Computer Science and Engineering, 1(2).

[3] Liang, C., Wu, J., Jian, H. Y., Deng, H. B., \& Wu, Z. H. Instant recommendation for web services composition. IEEE Transactions on Service Computing.

[4] Mansour, H. E., \& Dillon, T. (2011), Dependability and rollback recovery for composite web services. IEEE Transactions on Services Computing, 4(4).

[5] Andrea, Z., George, S., James, D., \& Igor, S. (2013), Proactive and reactive runtime service discovery: A framework and its evaluation, IEEE Transactions on Software Engineering, 39(7).

[6] Yang, X., \& Susan, D. U. (2012), IEEE computer society, using rules and data dependencies for the recovery of concurrent processes in a service-oriented environment. IEEE Transactions on Services Computing, 5(1).

[7] Qi, Y., \& Athman, B. (2013). Efficient service skyline computation for composite service selection. IEEE Transactions on Knowledge and Data Engineering, 25(4).

[8] Michael, P., \& Aphrodite, T. (2013). A generic query model for the unified discovery of heterogeneous services. IEEE Transactions on Services Computing, 6(2).

[9] Hua, C., Zhang, H. L., Zhao, W. B., Smith, P. M. M., Member, \& Louise, E. M. (2013). Toward trustworthy coordination of web services business activities. IEEE Transactions on Services Computing, 6(2). 
[10] Yesid, J., Keerthana, B., Marcelo, D. D. A., Yannis, V., \& Robert, D. C. Dynamic service contract enforcement in service-oriented networks, IEEE Transactions on Services Computing, 6(1).

[11] Aabhas, V. P., Basit, S., Jaideep, V., Hui Xiong, \& Nabil, A. (2012). Semantics-based automated service discovery. IEEE Transactions on Services Computing, 5(2).

[12] Lakshmi, S., Dinesh, K. S., \& Iaeng, T. V. P. (2013). Design of a recommender system for web based learning, Proceedings of the World Congress on Engineering.

[13] Mustapha, A., Mohamed, Q., Nicolas, D., \& Zahi, J. Web services discovery and recommendation based on information extraction and symbolic reputation. International Journal on Web Service Computing (IJWSC), 4(1).

[14] Pratheeba, R., \& Purushothaman, R. (2013). Modeling smarty web search engine using xml clustering. International Journal of Inventions in Computer Science and Engineering.

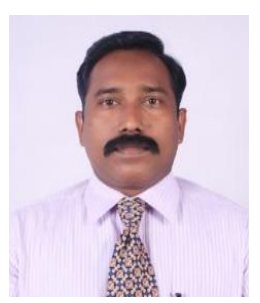

Muruganantham Balkrishnan obtained his bachelor degree in computer science and engineering from Manonmaniyam Sundaranar University Tirunelveli in 1994, and the master of technology in computer science and engineering from SRM University, in 2006. Currently he is working in SRM University and his area of interest is software engineering, distributed computing, database and data warehouse.

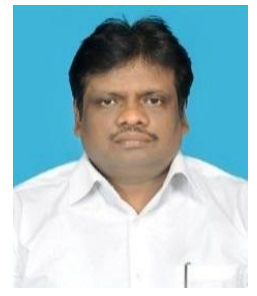

K. Vivekanandan obtained his B.E degree in electronics and communication engineering from Coimbatore Institute of Technology in 1986, the M. Tech in computer science and engineering from Indian Institute of Technology in 1991 and the Ph.D in 2005 from Pondicherry University in the area of software engineering. His teaching research and development interests have been in the areas of software engineering, object oriented systems, DBMS and data warehousing, information security and data mining. 